\title{
Influência do foco de atenção e intervalo de tempo na aprendizagem motora de crianças em idade escolar
}

\author{
Influence of the focus of attention and time interval the \\ motor learning in school children
}

\author{
Raphael Borges da Silva ${ }^{1}$, Yasmine Amad ${ }^{1}$, Cristina dos Santos Cardoso de \\ Sá $^{2}$
}

\begin{abstract}
1.Fisioterapeuta formado pela Universidade Federal de São Paulo, Campus Baixada Santista, Bolsista de iniciação científica CNPQ. Santos-SP, Brasil.

2.Fisioterapeuta. Profa. Dra. Associada do Curso de Fisioterapia Universidade Federal de São Paulo Campus Baixada Santista. Departamento de Ciências do Movimento Humano, Universidade Federal de São Paulo. Santos-SP, Brasil.
\end{abstract}

\begin{abstract}
Resumo
Introdução. Diversos fatores influenciam o processo de aprendizagem motora. 0 foco de atenção e a organização da prática são utilizados como estratégia de treinamento, de forma a instituir uma prática que leve ao melhor desempenho e retenção da tarefa treinada. Objetivo. Este estudo verificou a influência do intervalo de tempo e do foco de atenção na aquisição, transferência e retenção de uma tarefa motora. Método. Vinte crianças típicas entre 11 e 13 anos de ambos os gêneros foram divididas em 4 grupos (G1- $20 \mathrm{~s}$ e foco externo; G2- $20 \mathrm{~s}$ e foco interno; G3 - $60 \mathrm{~s}$ foco externo e G4 - $60 \mathrm{~s}$ foco interno). Os grupos foram submetidos ao teste pré-treino, 4 sessões de treino com 30 tentativas cada, testes de retenção imediata e transferência após 30 min do término da última sessão, e a teste de retenção após uma semana sem treinamento. Resultados. O G1 apresentou melhor desempenho durante a fase de aquisição. Houve retenção após o treinamento e isto independe do intervalo de tempo entre as tentativas e do foco de atenção, e todos os grupos mantiveram o desempenho após uma semana sem treino. Já para as tarefas de transferência, verificamos que independe do grupo e do tipo de foco de atenção, houve transferência da tarefa treinada. Conclusão. Concluímos que o intervalo de tempo entre as tentativas e tipo de foco de atenção interfere no desempenho durante a aquisição. Há retenção imediata e mesmo após uma semana sem treino, independente do grupo. Para todos, houve transferência da tarefa treinada.
\end{abstract}

Unitermos. Atenção; Aprendizagem motora; Habilidade motora; Criança

\begin{abstract}
Introduction. Several factors influence the process of motor learning. The focus of attention and organization of practice are used as a training strategy in order to establish a practice that leads to better performance and retention of trained task. Objective. This study verified the influence of the time interval and the focus of attention in the acquisition, transfer and retention of a motor task. Method. Twenty typical children between 11 and 13 years of both genders were divided into 4 groups (G1 - 20 s and external focus; G2 - 20 s and internal focus; G3 - $60 \mathrm{~s}$ external focus and G4 - $60 \mathrm{~s}$ internal focus). The groups were subjected to pretraining test, four training sessions of 30 trials each, ready retention and transfer tests after 30 min after the last session, and retention test after one week without training. Results. The G1 showed better performance during the acquisition phase.
\end{abstract}


There was retention after training and this is independent of the time interval between attempts and the focus of attention, and all groups maintained performance after a week without training. As for the transfer of tasks, we find that independent of the group and the type of focus of attention, there was transfer of the trained task. Conclusion. We concluded that the time interval between retries and type of attention focus interferes with performance during acquisition. There are immediate and even after a week without training retention, regardless of group. For all, there was transfer of the trained task.

Keywords. Attention; motor learning; motor skill; Child

Trabalho realizado na Universidade Federal de São Paulo, Campus Baixada Santista. SantosSP, Brasil.

Conflito de interesse: não Recebido em: 2018 Aceito em: 2018

Endereço para correspondência: Cristina SC Sá. Rua Silva Jardim 136, Departamento de Ciências do Movimento Humano. CEP 11015-020, Santos-SP, Brasil. cristina.sa@unifesp.com.br

\section{INTRODUÇÃO}

A aprendizagem motora pode ser definida pelas mudanças, associadas a prática ou a experiência, em processos internos que determinam a capacidade da pessoa de produzir certo movimento ${ }^{1}$. Este processo está relacionado com a alteração neural incluindo a estrutura e função de células nervosas e suas conexões, em diferentes regiões do sistema nervoso ${ }^{2,3}$. Segundo Maggil ${ }^{4}$ o aprendizado pode ser observado por meio de quatro características do desempenho: melhora ao longo do tempo, consistência entre as tentativas, persistência da habilidade por períodos mais longos de tempo e adaptação da habilidade para diferentes contextos.

Considerado um dos fatores que atuam sobre o desempenho motor, o foco de atenção pode ser definido como o direcionamento da atenção para características específicas em um ambiente de desempenho ou no planejamento da atividade ${ }^{4}$. O direcionamento do foco de 
atenção pode ser dividido entre interno e externo. O foco de atenção interno é definido como instruções ou feedback relacionados diretamente aos movimentos do próprio corpo do indivíduo. O foco externo está relacionado ao efeito dos movimentos corporais no ambiente ou no equipamento que está sendo utilizado ${ }^{5}$. De acordo com Wulf ${ }^{6}$ muitos estudos demonstraram que a precisão e a qualidade dos movimentos dependem expressivamente de onde o foco de atenção está enquanto uma atividade é executada.

Além do foco de atenção, outros fatores podem influenciar tanto no desempenho quanto no aprendizado da tarefa, entre eles a forma como o treino é organizado. Diversos fatores constituem a organização do treino e consequentemente a aquisição e retenção de tarefas motoras. A interferência contextual (IC) é um deles, sendo ela a interferência que resulta na variação da realização de uma tarefa dentro do contexto de prática ${ }^{4}$. Foi observado que em treinos com alta IC, apesar do indivíduo apresentar piores resultados durante a aquisição, seu desempenho nos testes de retenção e transferência da tarefa foi melhor ${ }^{4}$. Podemos concluir então que, apesar de prejudicar os resultados num contexto de prática essa estratégia melhora o desempenho durante os testes de retenção e transferência, o que mostra os benefícios de um treino com alta IC em comparação a um treino sem ou com baixa IC. Lee e Magill 7,8 argumentaram que 0 efeito de IC assemelha-se ao efeito observado pelo aumento do intervalo de tempo entre repetições que ocorre no 
aprendizado verbal. Propuseram ainda que o efeito de espaçamento das repetições (intervalo de tempo entre as tentativas) observado por Jacoby ${ }^{9}$ poderia explicar o efeito de IC no aprendizado motor. Dessa forma, treinar a mesma habilidade motora com diferentes intervalos de tempo entre as tentativas pode influenciar na aquisição e retenção da mesma ${ }^{10}$.

O aprendizado motor é muito mais visível durante a fase da infância e da adolescência, uma vez que é nesse momento que se adquire o controle postural e as habilidades motoras que iremos levar para a vida adulta. 0 período dos 6 aos 10 anos de idade marca a transição do refinamento das habilidades motoras fundamentais para as refinadas. Nesta idade, a maioria das habilidades motoras fundamentais tem potencial para estarem bem definidas, mas as atividades que envolvem os olhos e os membros desenvolvem-se lentamente. A partir dos 10 anos 0 comportamento motor esperado é caracterizado pela fase de habilidades motoras especializadas, ocorrendo mudanças na exatidão e no controle motor, porém não no padrão motor ${ }^{11}$.

A importância de avaliar a influência do foco de atenção e do intervalo de tempo na realização de atividades motoras simples é poder otimizar o aprendizado motor, tanto no contexto escolar e esportivo quanto na prática clínica, pois baseado nos achados é possível para o profissional identificar estratégias que possam facilitar o aprendizado motor em diferentes contextos. Frente ao 
exposto, o objetivo deste estudo foi verificar a influência do intervalo de tempo (20 e 60 segundos) entre as tentativas e do foco de atenção (interno e externo) na aquisição, transferência e retenção de uma tarefa motora em crianças em idade escolar.

\section{MÉTODO}

\section{Amostra}

O delineamento experimental deste estudo foi baseado no estudo de Sá $^{10}$ sobre Aquisição, Retenção e Transferência de Habilidades Motoras em Crianças de 7 e de 12 Anos. A amostra do nosso estudo contou com 20 crianças típicas de 11 a 13 anos, de ambos os gêneros, divididas em quatro grupos com cinco participantes cada pareados por idade e gênero. Sendo o número da amostra delimitado por conveniência.

Foram incluídas no estudo, crianças devidamente matriculadas em escolas do ensino fundamental com dominância de membros superiores à direita, não portadoras de qualquer deficiência física, cognitiva ou limitação do membro superior, cujos pais permitiram sua participação no estudo que foi aprovado pelo comitê de ética em pesquisa da instituição sob o número 674.575.

\section{Procedimento}

As crianças foram divididas em quatro grupos escolhidos por meio de sorteio. Todos os grupos 
executaram a mesma tarefa motora, diferenciando apenas as instruções com relação ao direcionamento do foco de atenção (interno ou externo) e ao intervalo de tempo entre as tentativas (20 ou $60 \mathrm{~s}$ ).

Para a execução da tarefa treinada (tarefa principal) foi utilizado um alvo de madeira com $1 \mathrm{~m}$ de diâmetro possuindo cinco circunferências concêntricas com raios de $10,20,30,40$ e $50 \mathrm{~cm}$ posicionado horizontalmente no chão plano a 2,5 $\mathrm{m}$ a frente do participante, contando a partir do centro do alvo (Figura 1). Cada circunferência recebeu um número, de 1 a 5 da periferia para o centro, para facilitar 0 registro do desempenho em cada tentativa. O alvo também foi dividido em nove setores radiais iguais de $40^{\circ}$ cada. Cada setor foi identificado com uma letra específica (de A a I), que juntamente com o número da circunferência indicou a localização exata do desempenho executado pelo participante. Foram utilizados no treinamento 30 saquinhos de areia, com peso de $50 \mathrm{~g}$, e 10 saquinhos com peso $100 \mathrm{~g}$ de peso nas tarefas de transferência 1 e 2 (TF1 e TF2, respectivamente). Os saquinhos foram embebidos em talco, para que fosse possível identificar a localização exata do arremesso no alvo.

Para o direcionamento do foco externo, as crianças foram orientadas para que no início de cada treino, prestar atenção no centro do alvo ao lançar o saquinho, visando acertar o mais próximo possível do centro, visto que, o direcionamento do foco externo visa buscar o impacto do movimento realizado sobre o ambiente ${ }^{12}$. Já para o grupo 
de foco interno, cada criança foi orientada no início de cada treino a prestar atenção no movimento do próprio braço ao lançar o saquinho de areia, visando acertar o mais próximo possível do centro do alvo. Cinco segundos antes do lançamento, a criança era orientada a fechar os olhos, recebendo os seguintes comandos verbais: "feche os olhos, concentre, pode jogar", pois, ao direcionar o participante para o foco interno, esta deve concentrar-se ao movimento de seu corpo para a realização da tarefa ${ }^{12}$.

Antes de iniciar as sessões de treino, cada criança realizou um pré-teste (PT) que consisti em cinco tentativas de lançamento de saquinhos de areia sem direcionamento do foco. Na sequência, foi realizada a etapa de treino, 4 sessões com 30 tentativas cada realizadas duas vezes na semana. Cada criança realizou o treino de acordo com seu grupo: G1- 20 s com o foco de atenção externo, G2- 20 s com foco de atenção interno, G3 - 60 s com foco de atenção externo e G4 - 60 s com foco de atenção interno. Aproximadamente na $15^{\mathrm{a}}$ tentativa o aplicador do teste redirecionava a criança relembrando o foco de atenção. Cada sessão de treino durou 30 minutos para os grupos com intervalo de tempo de $60 \mathrm{~s}$ e 10 minutos para os grupos com intervalo de tempo de 20 s.

Finalizada a fase de treino, foram feitos os testes de retenção 1 e 2 . Neste teste, a criança reproduziu a tarefa do treino, realizando cinco arremessos sem direcionamento do foco de atenção. O teste de retenção-1 foi realizado 30 minutos após a última sessão de treino e o teste de 
retenção-2 foi realizado uma semana após a última sessão de treino. $O$ teste de retenção durou aproximadamente 2 minutos para os grupos de intervalo 20 s e 5 minutos para 0 grupo com intervalo de tempo 60s.

Foram realizados duas tarefas de transferência. $\mathrm{Na}$ TF1 o participante estava posicionado a 3 metros de distância do alvo, e os saquinhos de areia tinham 100 gramas de peso. A TF1 foi realizada logo após o primeiro teste de retenção, 30 minutos após a última sessão de treino. O participante realizou 10 arremessos, respeitando o intervalo de tempo em que foi treinado. Na TF2 o participante estava posicionado a um ângulo de $45^{\circ}$ da posição original (Figura 1), e os saquinhos de areia tinham 100 gramas de peso. A TF2 foi realizada logo após a TF1. Novamente, o participante realizou 10 arremessos respeitando o intervalo de tempo em que foi treinado.
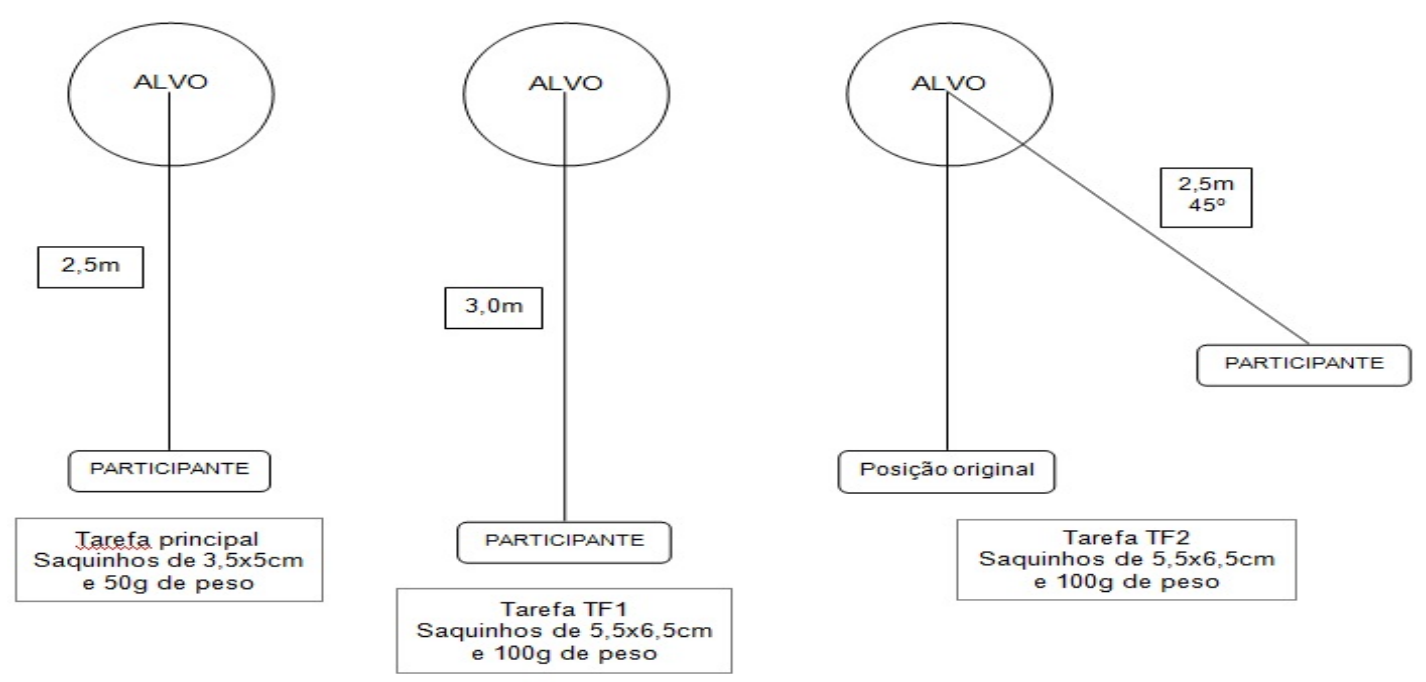

Figura 1. Esquema representativo da posição do participante e do alvo na tarefa principal, TF1 e TF2. 


\section{Análise Estatística}

A estratégia utilizada para a análise dos dados foi transformar os valores da excentricidade obtidos em cada arremesso executado pelo participante em medidas métricas (centímetros). Após esta transformação, para avaliar a evolução do desempenho ao longo do treinamento cada umas das sessões de treino (S1, S2, S3 e S4), cada qual contendo 30 tentativas, foi dividia em três blocos de 10 tentativas. Calculou-se a mediana de cada bloco de 10 tentativas para cada participante e, a partir desses escores, a média das medianas de cada grupo em cada um dos blocos de cada sessão.

Para avaliar a retenção da tarefa treinada, calculouse a média de cada participante no teste de avaliação prétreinamento e para os testes de retenção 1 e 2, e, em seguida a mediana das médias para cada grupo. Calculouse também as medianas de cada participante nas tarefas de transferência 1 e 2 .

Comparando-se a natureza do teste utilizado, denomina-se "desempenho" a distância entre o centro do alvo e o local do alvo onde o saquinho de areia arremessado pelo participante 0 tocou no $1^{0}$ contato. Assim, enquanto "melhor desempenho" ou "desempenho superior" indica que essa distância foi relativamente menor, e "pior desempenho" ou "desempenho inferior" indica que essa distância foi relativamente maior.

A análise de variância para medidas repetidas (ANOVA) foi aplicada para os grupos de crianças treinadas 
com intervalo de 20 e 60 segundos, i.e. uma análise para cada intervalos de tempo entre as tentativas, tendo como fatores entre sujeitos o foco de atenção externo e interno, e como fator intra-sujeitos a repetição das sessões incluindo o teste de avaliação pré-teste e os testes de retenção 1 e 2, as quatro sessões de treino, cada qual com 3 blocos de 10 tentativas, e o último bloco das sessões de treino em relação as tarefas de transferências 1 e 2 . Em cada caso, quando houve diferenças significantes no fator principal, foram realizadas análise de contrastes post hoc para identificar a origem das diferenças, utilizando o teste de Student Newman-Keuls.

O nível de significância foi de $p<0,05$, e foi utilizado o programa Statistica para realizar as análises.

\section{RESULTADOS}

Os resultados do desempenho dos diferentes grupos, em termos das distâncias que os saquinhos de areia atingiram o centro do alvo nas sessões de treino, são apresentados na Figura 2 (note que quanto menor a distância melhor o desempenho). Os intervalos de tempo entre as tentativas interferem no desempenho das crianças $(p<0,0003)$, assim como o tipo de foco de atenção $(p<0,0004)$, e há interação entre o intervalo de tempo, entre as tentativas e o tipo de foco de atenção $(p<0,04)$. A análise post hoc revelou que o desempenho foi melhor no grupo que treinou com intervalo de $20 \mathrm{~s}$ e foco externo ( $\mathrm{G}$ 1) (Figura 2). 


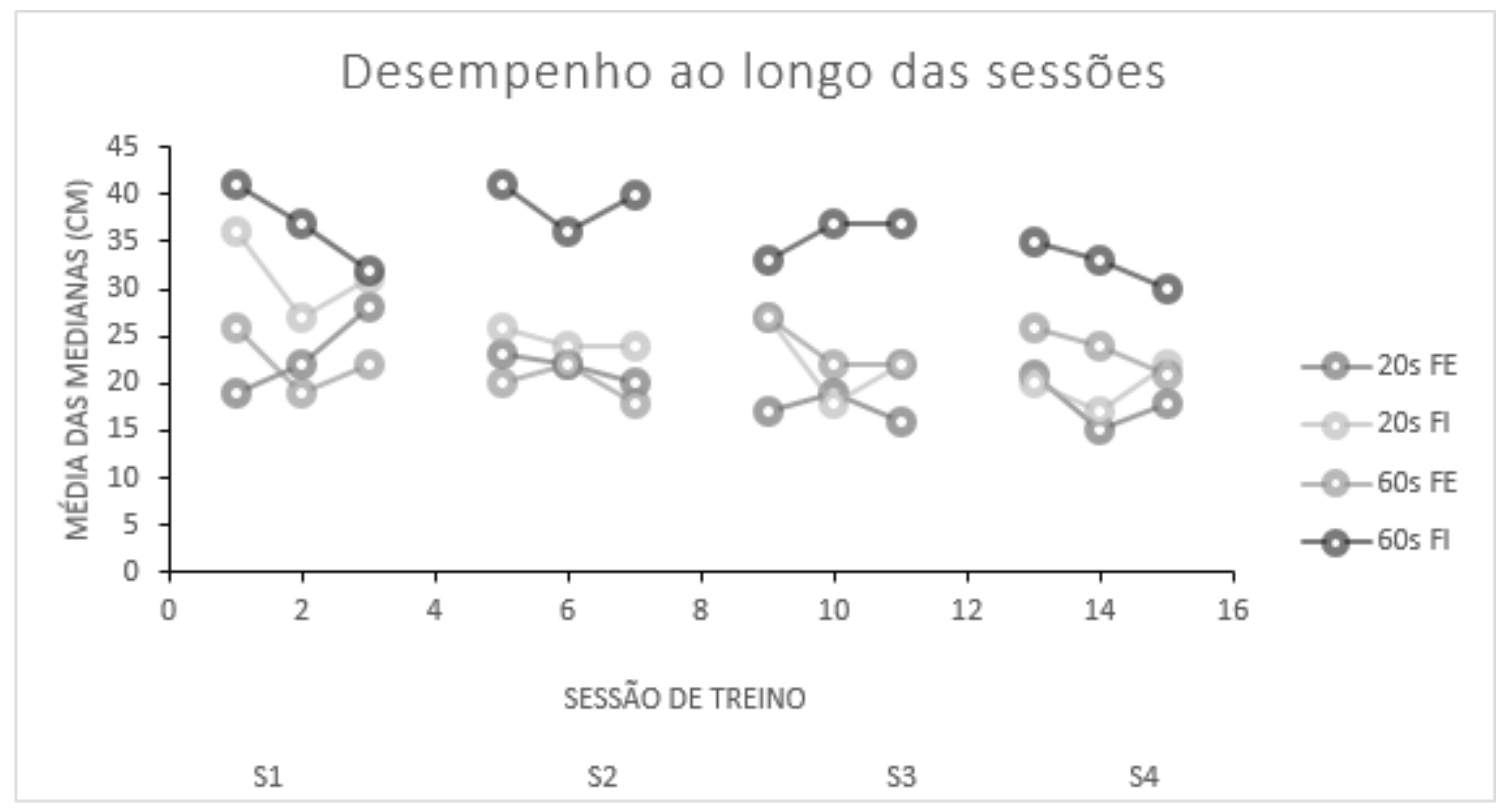

Figura 2. Média das medianas (em $\mathrm{cm}$ ) dos escores obtidos pelos participantes dos grupos em cada bloco de tentativas $(1,2,3)$ de cada sessão de treinamento (S1, S2, S3, S4) considerando o intervalo de tempo entre as tentativas (20 ou $60 \mathrm{~s}$ ), e o tipo de foco de atenção (FE- foco externo; FI - foco interno) (G1 -20s FE; G2 -20 s FI; G3 - 60 s FE; G4 - 60 s FI).

Os dados obtidos nos testes realizados no pré-teste (PT) realizado antes das sessões de treino (fase de aquisição) são demonstrados na Figura 3, associados aos dados obtidos na tarefa feita imediatamente após o termino da última sessão de treino (RT1) e os dados do teste feito uma semana após o termino do treinamento (RT2). Houve retenção após o treinamento $(p<0,001)$, e isto independe do intervalo de tempo entre as tentativas $(p<1,0)$ e do foco de atenção $(p<0,48)$, e para todos os grupos 0 desempenho se manteve após uma semana sem treinamento (Figura 3). 


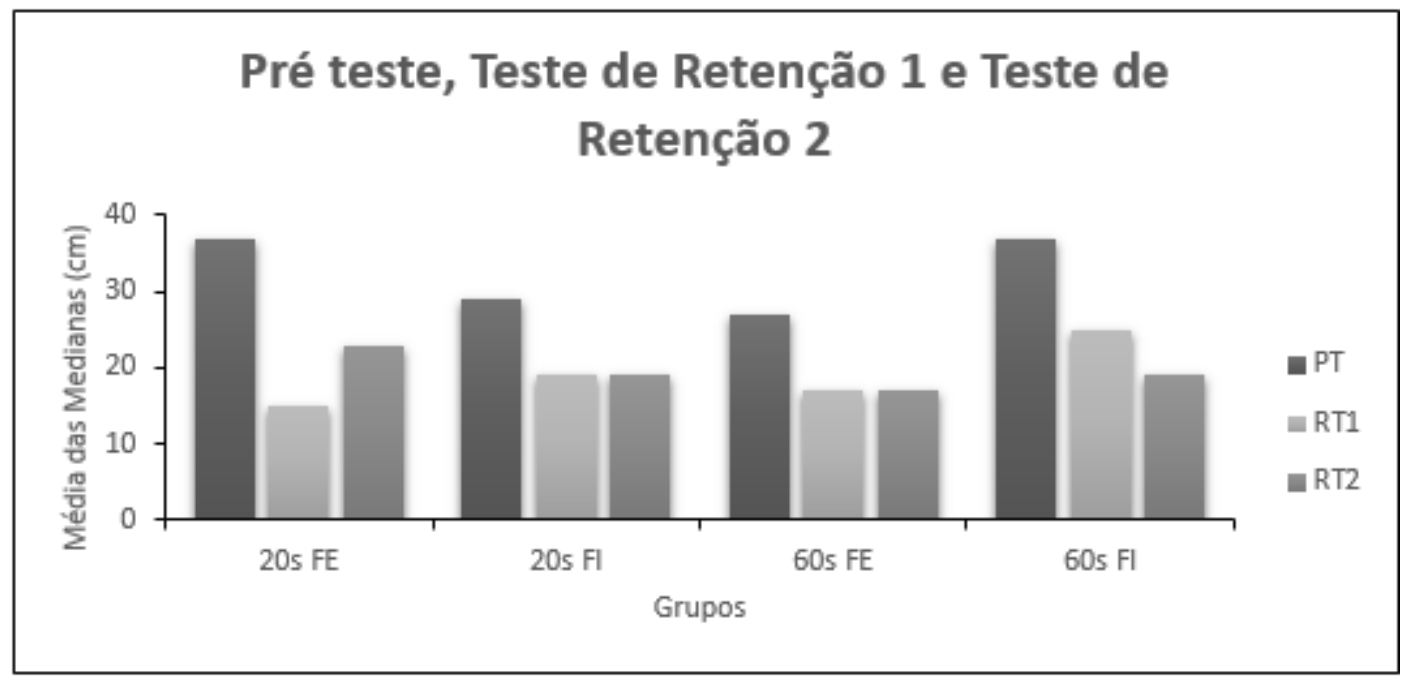

Figura 3. Média das medianas $(e \mathrm{~m} \mathrm{~cm}$ ) dos escores obtidos pelos participantes dos grupos nos testes de avaliação pré-treinamento (pré-teste (PT), teste de avaliação

pós-treinamento (TR1) e teste de avaliação pós- treinamento (TR2), (FE- foco externo; FI - foco interno) (G1 -20s FE; G2 -20 s FI; G3 - 60 s FE; G4 - 60 s FI).

Os dados obtidos nas TF1 e TF2 foram associados (Figura 4) aos dados do último bloco de 10 tentativas da última sessão de treino (BI3S4) de todos os grupos. Independente do grupo e do tipo de foco de atenção houve transferência da tarefa treinada $(p<0,000)$ (Figura 4).

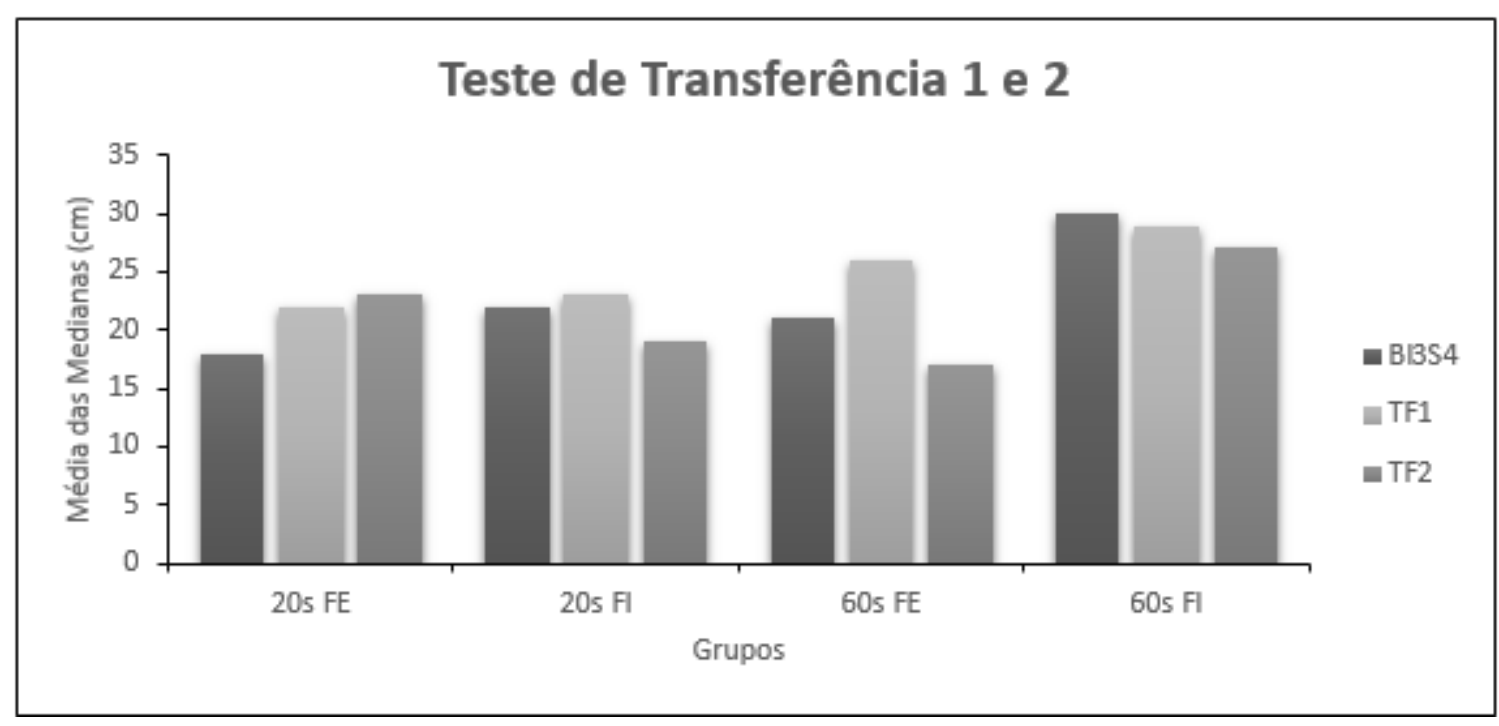

Figura 4. Média das medianas (em $\mathrm{cm}$ ) dos escores obtidos pelos participantes dos grupos no último bloco da última sessão de treino (BI3S4) e nas Tarefas de Transferência 1 e 2 (TF1 e TF2), (FE- foco externo; FI - foco interno) (G1 -20s FE; G2 -20 s FI; G3 - 60 s F FE; G4 - 60 s FI). 


\section{DISCUSSÃO}

Os resultados obtidos no estudo revelaram que os grupos treinados com o foco de atenção externo (FE - G1 e G3) se sobressaíram durante a fase de aquisição (sessões de treino) quando comparados aos os grupos treinados com o foco de atenção interno (FI - G2 e G4). Esse achado condiz com o encontrado por Wulf, McNevin e Shea ${ }^{13}$, que ao utilizarem $\circ \mathrm{FI}$, demonstraram que o praticante pode inibir ou interferir nos processos de controle automático do sistema motor que normalmente regulam o movimento, enquanto que o uso do FE permite que o sistema motor se organize de forma mais natural favorecendo seu desempenho. Outros estudos também suportam a teoria de que o FE resulta em melhor desempenho da tarefa, corroborando com os resultados obtidos ${ }^{14-17}$.

Ainda na fase de aquisição era esperado que os grupos com menor intervalo de tempo (20s) apresentariam melhor desempenho, pois, o intervalo mais curto entre as tentativas permitiria que o programa motor permanecesse mais ativo na memória operacional, dessa forma não seria necessário a reconstrução a cada tentativa ${ }^{10}$. Esta teoria suporta os resultados obtidos em nosso estudo, pois os grupos com menor intervalo de tempo tiveram melhor resultado nesta fase.

Silva et al. ${ }^{18}$ compararam 0 desempenho entre 5 grupos na tarefa de lançamento de dardos: FI, FE, foco interno-externo, foco externo-interno e grupo controle. Os resultados sugerem que o FI auxilia os praticantes no início 
da aprendizagem, pois estão mais focados nos aspectos cognitivos da tarefa, e que o FE é melhor após um período de prática, estes resultados indicam que o foco de atenção pode mudar conforme o nível de aprendizagem progride. No presente estudo os grupos treinados com FE apresentaram desempenho superior comparado aos grupos com FI, o que pode indicar que a tarefa imposta não tenha sido complexa ou inédita, sendo que as crianças possam já ter adquirido um padrão motor semelhante no dia-a-dia, previamente ao treinamento, e só aperfeiçoaram suas habilidades ao longo do treinamento.

Um estudo fez um comparativo da aquisição e retenção de habilidades treinadas em diferentes idades (crianças de 5 a 6 anos, de 7 a 8 anos e adultos de 19 a 29 anos), todos os participantes foram submetidos à diferentes tarefas e testes de movimentos de mão, nele todas as idades apresentaram retenção semelhante, entretanto crianças mais velhas e jovens adultos apresentaram platô de desempenho e velocidade de aprendizagem, já as mais novas puderam melhorar continuamente seu desempenho ${ }^{19}$. Se compararmos com as crianças de nosso estudo, podemos dizer que elas se assemelham, em relação ao desenvolvimento neuromotor, aos grupos mais velhos do estudo citado. Desta forma podemos supor que as crianças atingiram seu platô de velocidade para aprendizagem e ganho de precisão, o que necessitaria maior tempo de treino para que houvesse maior diferenciação nos resultados entre os grupos. 
Era esperado que os grupos que realizaram 0 treinamento com intervalo de tempo de 60s entre as tentativas obtivessem melhor desempenho nos testes de retenção comparados aos grupos treinados com intervalo de tempo de 20s. A hipótese era de que ao estipular intervalos de tempo maiores entre as tentativas, ocorre um decaimento do programa motor na memória operacional, o que leva o praticante a reconstruir o plano de ação a cada tentativa, gerando dessa forma maior retenção, decorrente do maior esforço cognitivo ${ }^{10}$. Entretanto, não houve diferenças nos resultados de retenção quando comparado aos grupos com menor intervalo de tempo (20s), uma nova analise com maior número de participantes seria necessária para confirmar os resultados.

Uma explicação para não termos conseguido constatar diferença na retenção da tarefa entre os grupos é que, aqueles participantes que deveriam utilizar o FI e tiveram maior intervalo de tempo (FI 60s - G4) entre as tarefas não se atentaram corretamente às dicas fornecidas (feche os olhos, concentre, pode jogar), desta forma tiveram os estágios de aprendizagem ${ }^{20}$ danificados, prejudicando 0 processo de atenção seletiva.

Fatores ambientais como, ausência de ambiente controlado sem interferências visuais e sonoras durante as atividades, e intrapessoais podem ter influenciado nestes resultados. Foi observado durante os treinos que algumas crianças participantes do nosso estudo apresentaram maior dificuldade em manter a concentração durante o treino, 
especialmente as que pertenciam ao grupo de 60s entre as tentativas. As mais agitadas acabavam dispersando entre as tentativas, 0 que pode ter influenciado em seu desempenho. Em outro estudo, a tarefa foi realizada em um ambiente controlado, silencioso, sem fatores que pudessem distrair os participantes ${ }^{15}$.

A análise do pré-teste, realizado antes do início do treino, e os testes de retenção de 30 minutos após a última sessão de treino e uma semana após a última semana de treino, não revelou grande diferença entre os grupos em relação ao intervalo de tempo e ao foco de atenção, porém, quando associados estes fatores apresentaram diferença significante entre o pré-teste, TR1 e TR2, ou seja, quando comparamos o desempenho obtido nos testes pós-treino com o teste realizado pré-treino, observamos que em todos os grupos os participantes melhoraram o desempenho após o treino, indicando que independente da estratégia utilizada, o treino leva a melhora no desempenho.

Ao observar que não existiram diferenças nos escores obtidos no TR1 e TR2, podemos concluir que o aprendizado se manteve relativamente estável após uma semana sem treino, o que pode ser explicado pois esquemas motores tornam-se progressivamente mais estáveis e resistentes à interferência com a prática, revelando um processo de consolidação da memória que acontece mesmo após a prática real tenha terminado, chamados período off-line ${ }^{21}$.

Os resultados das tarefas de TF1 e TF2 não demonstraram variação em relação aos fatores intervalo de 
tempo e foco de atenção. Com base nos estudos encontrados na literatura $0 \mathrm{FE}^{13}$ e o maior intervalo de tempo entre as tentativas $^{10}$ resultam em melhor desempenho na tarefa de transferência. Como pode ser visto na Figura 4, houve transferência da tarefa treinada independente do tipo foco de atenção e intervalo de tempo adotado.

Uma possível justificativa para os resultados das tarefas de transferência é que ao aumentar a distância do alvo exigiu-se que os voluntários investissem mais força no arremesso. Ao imprimir uma força maior geralmente prejudica-se a precisão ${ }^{22}$. Os resultados obtidos na tarefa de transferência não são devidos à falta de aprendizagem, mas sim na adaptação física da tarefa, ou seja, as crianças foram capazes de transferir a tarefa treinada, entretanto não foram capazes de manter o desempenho da fase de aquisição, perdendo precisão para acertar o centro do alvo $^{15}$.

Seria possível, e de que forma, uma prática ou treino afetariam no desenvolvimento da função executiva na infância, e nos apresenta vários programas propostos para impactar positivamente na função executiva, sendo eles baseados em evidencia cientifica ou com pouca base empírica, porém com grande aceitação entre os educadores e pais ${ }^{23}$. Ao final, aponta que estratégias com componentes de prática ativa podem ter melhor resultado no aperfeiçoamento da função executiva. Ao confrontarmos este questionamento com os resultados obtidos em nosso 
estudo e com o que é encontrado na literatura com relação à adoção de estratégias de treino para aquisição e aprimoramento de habilidades motoras em crianças, podemos afirmar que a pratica de uma atividade utilizando o tipo de foco de atenção ideal para cada momento do processo de aprendizagem, respeitando o intervalo de tempo necessário para que se crie um padrão de movimento não apenas mecânico, mas consolidado na memória operacional, pode interferir na função executiva de forma positiva impactando no aprimoramento e melhor aproveitamento destas atividades.

\section{CONCLUSÃO}

Os resultados do presente estudo apontam que os grupos treinados com 0 foco de atenção externo apresentaram melhor desempenho que os grupos treinados com foco de atenção interno, e que o intervalo de tempo entre as tentativas interfere no desempenho das crianças, assim como o tipo de foco de atenção. Há interação entre os fatores foco de atenção e intervalo de tempo, e o grupo treinado com menor intervalo de tempo e foco externo (G1) apresentou melhor resultado durante a fase de aquisição. Há retenção após o treinamento, e isto independe do intervalo de tempo entre as tentativas e do foco de atenção adotado, e que em todos os grupos o desempenho se manteve após uma semana de treino. Na tarefa de transferência, concluímos que houve transferência da tarefa 
treinada, e isto independe do tipo de foco de atenção utilizado e do intervalo de tempo entre as tentativas.

\section{REREFÊNCIAS}

1.Schmidt RA, Wrisberg CA. Motor Learning and Performance: A Situation-based Learning Approach, 4. ed. Champaign: Human Kinetics, 2008, 426p.

2.Kandel ER, Schwartz JH, Jessel TM. Essentials of Neural Science and Behaviour. Nova Jersey: Appleton \& Lange, 1995.

3.Kandel ER, Schwartz JH, Jessel TM. Principles of Neural Science and Behaviour, 4.ed. Nova Jersey: Appleton \& Lange, 2000.

4.Magill RA. Motor Learning and Control: Concepts and Applications. 7. ed. Boston: McGraw-Hill, 2004.

5.Wulf $G$, $H o ̈ ß ~ M$, Prinz W. Instructions for Motor Learning: Differential Effects of Internal Versus External Focus of Attention. J Mot Behav 1998;30:169-79.

http://dx.doi.org/10.1080/00222899809601334

6. Wulf G. Attentional focus and motor learning: a review of 10 years of research. E-J Bewegung Training 2007;1:4-14.

7.Lee TD, Magill RA. The locus of contextual interference in motor skill acquisition. J Exp Psychol: Learning, Memory and Cognition. 1983;9:730-46. http://dx.doi.org/10.1037/0278-7393.9.4.730

8.Lee TD, Magill RA. Can forgetting facilitate skill acquisition? In: Goodman D, Wilberg RB, Franks JM. Differing perspective in motor learning, memory and control. Amsterdan: North Holland, 1985; 322.

9.Jacoby LL. On interpreting the effects of repetition: Solving a problem versus remembering a solution. J Verbal Learn Verbal Beh 1978; 17:649-66. https://doi.org/10.1016/S0022-5371(78)90393-6

10.Sá CSC, Xavier GF. Aquisição, retenção e transferência de habilidades motoras em crianças. Saarbrucken: Novas Edições Acadêmicas, 2016.

11.Aandrade A, Luft CB, Rolim MKS. O desenvolvimento motor, a maturação das áreas corticais e a atenção na aprendizagem motora. Buenos Aires: Revista Digital 2004;78:14p. http://www.efdeportes.com/efd78/motor.htm

12.Jarus T, Ghanouni P, Abel RL, Fomenoff SL, Lundberg J, Davidson $S$, et al. Effect of internal versus external focus of attention on implicit motor learning in children with developmental coordination disorder. Res Dev Disabil 2015;37:119-26. https://doi.org/10.1016/j.ridd.2014.11.009

13. Wulf G, Mcnevin N, Shea C. The automaticity of complex motor skill learning as a function of attentional focus. J Exp Psychol 2001;54:1143-54. https://doi.org/10.1080/713756012 
14.Wulf G, Shea C, Park J. Attention and motor performance: Preferences for and advantages of an external focus. Res Quarterly Exer Sport https://doi.org/10.1080/02701367.2001.10608970

15.Emanuel M, Jarus T, Bart O. Effect of Focus of Attention and Age on Motor Acquisition, Retention, and Transfer: A Randomized Trial. Phis Therp. 2008;88:251-60. https://doi.org/10.2522/ptj.20060174 16.Zentgraf $\mathrm{K}$, Munzert J. Effects of attentional-focus instructions on movement kinematics. Psychol Sport Exer 2009;10:520-5.

17. Lohse KR, Sherwood DE, Healy AF. How changing the focus of attention affects performance, kinematics, and electromyography in dart throwing. Hum Mov Sci 2010;29:542-55. https://doi.org/10.1016/j.humov.2010.05.001

18.Silva CBM, Benda RN, Fonseca FS, Fialho JVAP, Menzel HJK, Ugrinowitsch $\mathrm{H}$. Mudança no foco de atenção ao longo da prática de uma habilidade motora. Motriz 2013;19:391-8.

19.Julius MS, Adi-Japha E. Learning of a simple grapho-motor task by Young children and adults: similar acquisition but age-dependent retention. Front Psychol 2015;6:225. https://doi.org/10.3389/fpsyg.2015.00225

20.Fitts PM, Posner MI. Human performance. Belmont: Brooks/Colemann, 1967.

21.Krakauer JW, Shadmehr R. Consolidation of motor memory. Trends Neurosci 2006;29:58-64. https://doi.org/10.1016/j.tins.2005.10.003

22. Etnyre RB. Accuracy characteristics of throwing as a result of maximum force effort. Perc Mot Skillhs 1998;86:1211-7. https://doi.org/10.2466/pms.1998.86.3c.1211

23.Shaheen S. How child's play impacts executive function-related behaviors. Appl Neuropsychol Child 2014;3:182-7. https://doi.org/10.1080/21622965.2013.839612 